\title{
Eficácia do sistema de contenção (automatizado e mecânico) no atordoamento de bovinos
}

\author{
Effectiveness of the restraining system (automated and mechanical) in the cattle stunning
}

\author{
William Bertoloni ${ }^{I}$ Douglas Andreolla II
}

\section{RESUMO}

Um total de 800 bovinos machos (nelore) anelorados), com idade entre 28 e 36 meses, foi atordoado com pistola pneumática, com pressão de operação de 11 a 12 bar e haste de penetração de $15.9 \mathrm{~mm}$ de diâmetro, em sistemas de contenção diferentes (mecânico e atomatizado). $O$ box de contenção mecânico tradicional $(2,53 \times 0,88 \times 2,20 \mathrm{~m})$ não permitiu a contenção da cabeça do animal; no segundo tratamento utilizou-se box de contenção automatizado $(2,60 \times 0,85 \times 2,30 m)$, com parede lateral e piso móveis, guilhotina e elevador de cabeça. O box automatizado proporcionou menor número de disparos necessários para o atordoamento $(1,0625 \pm 0,013)$ em comparação ao mecânico(1,2025 $\pm 0,026)$, ( $P \leq 0,0001)$, reduziu a distância em relação ao ponto ideal no primeiro disparo $(1,246 \pm 0,918 e$ $2,357 \pm 1,246)$ e no segundo $(3,042 \pm 0,141$ e 4,016 $\pm 0,101)$, $(P \leq 0,0001)$, assim como proporcionou ausência de reflexo respiratório comparativamente ao sistema mecânico $(0,0275 \pm 0,008),(P \leq 0,001)$. O box automatizado apresentou maior bem-estar e maior eficácia na contenção $e$ insensibilização dos bovinos

Palavras-chave: estresse, sistema de atordoamento, abate, bem-estar.

\section{ABSTRACT}

A total of 800 male cattle (Nelore), aged 28-36 months, stunned by pneumatic captive bolt pistol (operating pressure 160-175psi 11-12bar, air consumption (per cycle) $1.45 \mathrm{ft}^{3} 41 \mathrm{~L}$, penetrating shaft diameter 0.625 in $15.9 \mathrm{~mm}$ ) in different restrainer systems (mechanical and automated). The traditional mechanical restraint $(2.53 \times 0.88 \times 2.20 \mathrm{~m})$ did not allow the containment of the animal's head, the automated restrainer $(2.60 \times 0.85 \times 2.30 \mathrm{~m})$ allowed adjustable sidewall and restraint and elevating the head. The automatic restrainer provided smaller number of shots required for stunning $(1.0625 \pm 0.013)$ compared to mechanical $(1.2025 \pm 0.026)$ $(P \leq 0.0001)$, reduced the distance from the ideal point in the first shot $(1.246 \pm 0.918)$ and $(2.357 \pm 1.246)$ and second (3.042 \pm 0.141$)$ and $(4.016 \pm 0.101)(P \leq 0.0001)$, and provided no respiratory reflex compared to the mechanical system (0.0275 \pm 0.008$),(P \leq 0.001)$. The automatic restrainer had a higher welfare, improving the efficiency of restraint and stunning of cattle.

Key words: stress, stunning system, slaughter, welfare.

\section{INTRODUÇÃO}

Para se realizar uma insensibilização eficaz, é importante que o animal esteja suficientemente imobilizado para se efetuar o disparo na posição correta, por isso os bovinos geralmente são imobilizados em equipamentos de contenção (box) mecânicos ou automatizados, dispondo de piso antiderrapante e espaço restrito, o que impede a movimentação e proporciona a insensibilização individual (GRANDIN, 2010).

HEDRICK et al. (1994) alertam para a influência exercida pelo tipo e pela eficiência dos processos de imobilização, afirmando que o uso inadequado desses equipamentos refletem

IDepartamento de Zootecnia e Extensão Rural (DZER), Faculdade de Agronomia e Medicina Veterinária (FAMEV), Universidade Federal de Mato Grosso (UFMT). Av. Fernando Corrêa, s/nº, Coxipó, 78060-900, Cuiabá, MT, Brasil. E-mail: bt.william@ig.com.br. Autor para correspondência.

IIPrograma de Pós-graduação em Ciência Animal, FAMEV, UFMT, Cuiabá, MT, Brasil. 
sensivelmente o grau de esgotamento do glicogênio muscular. Tais contrastes no nível de glicogênio resultam em diferenças no $\mathrm{pH}$ final e nas propriedades físico-químicas do tecido muscular, assim como na qualidade da carne.

O box de atordoamento com contenção da cabeça, uma alternativa para a melhoria no bem-estar e na qualidade da carne, limita o movimento do animal para frente, para trás e para os lados, impedindo dessa forma o movimento da cabeça e do corpo, o que proporciona maior precisão do disparo com pistola, com embolo penetrante ou não penetrante (H.S.A., 1998). Em alguns países, a utilização do box de contenção como imobilização da cabeça é obrigatória por lei como, por exemplo, na Inglaterra, quando se utiliza o abate com pistola de dardo cativo.

Embora a utilização de box de insensibilização com contenção de cabeça proporcione maior segurança e facilidade para o operador realizar o disparo na posição correta do crânio, quando utilizado erroneamente, poderá proporcionar um resultado negativo em comparação ao box convencional, visto que, se $o$ animal ficar muito tempo com a cabeça contida, poderá ocorrer uma elevação do nível de estresse e prejuízos na qualidade da carcaça (GRANDIN, 1996).

Atualmente, no Brasil, o método de insensibilização mais utilizado para bovinos é a pistola pneumática com embolo penetrante, a qual produz uma grave laceração encefálica, promovendo inconsciência rápida do animal (ROÇA, 1999). Entretanto, se sua utilização for inadequada, resultando em mais de um disparo para levar o animal à inconsciência, há prejuízos ao bem-estar do animal, que sente dor e tem seu nível de estresse aumentado, o que pode causar queda na qualidade da carne (CIOCCA et al., 2006).

As pistolas pneumáticas de penetração fabricadas no Brasil geralmente possuem terminal em bastão de $11 \mathrm{~mm}$ de diâmetro, com extremidade convexa e força de impacto de 8 a $12 \mathrm{~kg} \mathrm{~cm}^{-2}$. Não possuem injeção direta de ar com o objetivo de lacerar o tecido cerebral (PRATA \& FUKUDA, 2001).

Uma insensibilização efetiva com a pistola de dardo cativo depende da força do projétil e de que o golpe seja efetuado na parte correta do crânio. A melhor posição é onde o cérebro está mais próximo da superfície da cabeça, ou seja, local onde o crânio (osso frontal) é mais delgado; no bovino, a posição ideal corresponde à metade do osso frontal, onde se cruzam duas linhas imaginárias traçadas do centro da base dos cornos ao olho oposto (GALLO et al., 2003), proporcionando que o dardo atinja o cérebro de maneira mais fácil (EIKELENBOOM, 1983; H.S.A., 1995). Não se deve apontar entre os olhos ou atrás da cabeça porque é menos eficaz que a posição sobre o osso frontal (GRANDIN, 1994).

GALLO \& CARTES (2000) observaram que $7,3 \%$ dos bovinos contidos em box convencional e atordoados com pistola de embolo penetrante receberam disparo no espaço atlanto-oocipital (8cm do local ideal), atingindo o bulbo cerebral. Após a mudança para o box automatizado, observou-se uma redução para 4,3\% dos bovinos com disparo nessa posição.

Para um bom atordoamento, não apenas a posição do disparo é importante, mas também a angulação da pistola com o crânio do animal, devendo essa angulação permitir que o dardo ou a força de impacto atinja as principais estruturas cerebrais responsáveis pela inconsciência do animal, sendo elas: córtex cerebral, tronco encefálico e cerebelo (NEVES, 2008; FINNIE, 1993). Desse modo, a pistola deverá ser aplicada em ângulo reto em relação ao crânio para que o dardo tenha como destino o córtex cerebral em direção ao tronco cerebral (EIKELENBOOM, 1983; H.S.A., 1995; 1998).

A H.S.A. (1995) pondera que, se nos bovinos a penetração do dardo cativo estiver entre 4$6 \mathrm{~cm}$ do local ideal, a eficácia do disparo somente chega a $60 \%$. Segundo GREGORY et al. (2007), quando a posição do disparo dista mais de $2 \mathrm{~cm}$ do local ideal de disparo, aumenta-se o risco de uma insensibilização mal feita ou de o animal retornar mais rápido à consciência. O número de disparos está associado à posição do primeiro disparo, visto que, se o primeiro disparo for realizado em um diâmetro de até $2 \mathrm{~cm}$ do ponto ideal, a necessidade de novos disparos fica muito reduzida.

Porém, resultados apresentados por GRANDIN (2002) demonstraram que, quando a insensibilização é feita com a pistola de dardo cativo com penetração, essa distância pode chegar até $6 \mathrm{~cm}$, sem interferir na qualidade da insensibilização, pois a efetividade do disparo vai depender mais da direção e da força com que se realiza o disparo do que do lugar preciso de penetração.

Na figura 1, é apresentado um desenho que mostra claramente a importância da angulação da pistola no atordoamento dos bovinos. A letra A indica o posicionamento correto e a angulação correta do disparo, e as letras B e C demonstram o posicionamento correto da pistola, porém com angulações inadequadas, não atingindo as estruturas encefálicas que garantem o bom atordoamento (NEVES, 2008).

$\mathrm{O}$ atordoamento de bovinos pelo método de pistola de dardo cativo de penetração tem como princípio um forte impacto no cérebro, de modo a causar uma disfunção da atividade elétrica normal, em razão 


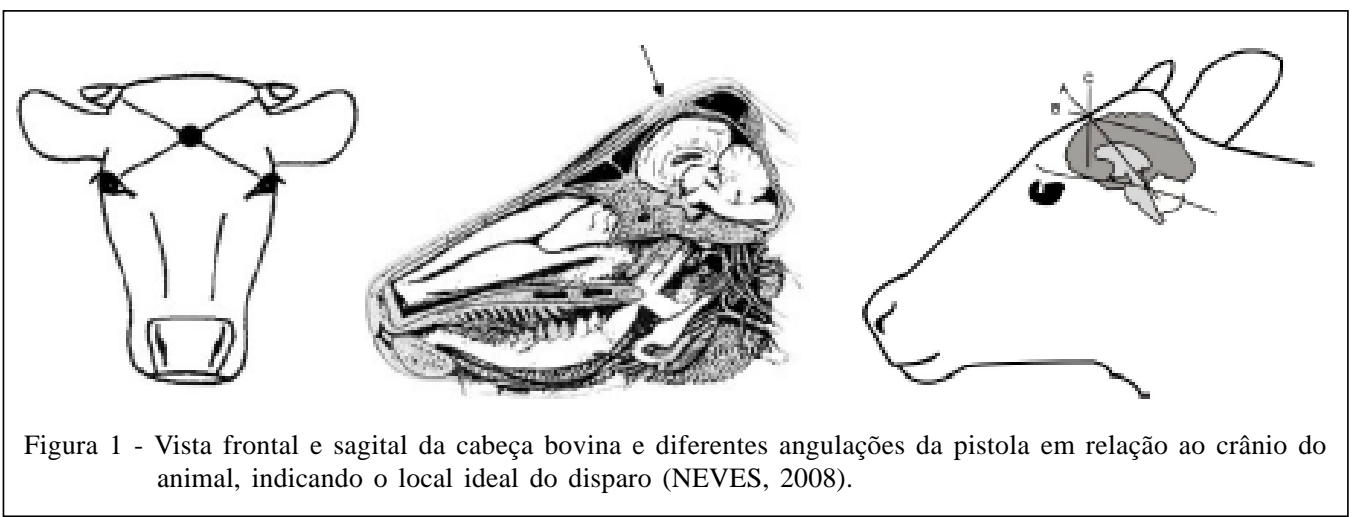

de uma dramática mudança de pressão (ROÇA, 2002). As lesões cranianas encontradas no abate com pistola de dardo cativo com penetração são bem discretas, não havendo, na maioria das vezes, fratura do crânio e sim uma perfuração de aproximadamente um cm de diâmetro. A entrada do dardo cativo no encéfalo provoca uma grande, profunda e bem definida hemorragia em seu trajeto, com severa destruição e perda de tecido neural do cerebelo e mesencéfalo, atingindo frequentemente a ponte, a medula oblonga e a parte caudal do córtex cerebral (FINNIE, 1993).

Pela importância do tema e pequeno número de pesquisas realizadas sobre o assunto, objetivou-se avaliar a influência do sistema de contenção automatizado e mecânico no bem-estar de bovinos insensibilizados com pistola pneumática penetrante.

\section{MATERIAL E MÉTODOS}

Oitocentos bovinos, machos (nelore/ anelorados), com idade entre 28 e 36 meses, subdivididos em dois blocos de 400 animais (100 bovinos no início das atividades do abatedouro, 100 no final do período da manhã, 100 no início da tarde e 100 no final da tarde de cada bloco), foram abatidos em abatedouro-frigorífico com capacidade para 120 animas hora $^{-1}$, na cidade de Várzea Grande, Mato Grosso (MT).

Foram utilizados dois sistemas de contenção (box) para o atordoamento, com unidades geminadas, construídas em contiguidade imediata à fila indiana e intercomunicada através de portas em guilhotina, sendo comumente encontrados no mercado.

O primeiro sistema de contenção (box 1$)$ era mecanizado e possuía 2,53m de comprimento, 0,88m de largura e 2,20m de altura, portão tipo guilhotina com fundo flanco e piso móveis. O segundo sistema de contenção (box 2) era automatizado e possuía 2,60m de comprimento, 0,85m de largura e 2,30m de altura, fundo, flanco e piso móveis, pescoceira e bandeja do tipo elevador de cabeça, o que proporcionava a imobilização completa e elevação da cabeça do animal.

Todos os animais foram atordoados com a mesma pistola pneumática, acionada por ar comprimido com pressão de operação (máxima) 160-175psi /1112bar, consumo de ar (por ciclo) de $1.45 \mathrm{ft}^{3} 41 \mathrm{~L}$, diâmetro da haste de atordoamento de $15,9 \mathrm{~mm}$ e peso de $14,7 \mathrm{~kg}$. A pistola era operada pelo mesmo funcionário.

Após o atordoamento dos animais, a eficácia do sistema de contenção associado ao sistema de insensibilização penetrante foi avaliada segundo GRANDIN (1999), com base nos seguintes critérios: eficiência do primeiro disparo no osso frontal independentemente de sua localização; número de disparos necessários para a queda no box de atordoamento; distância entre o local do disparo e o ponto da convergência entre duas linhas imaginárias oriundas da base do chifre ao globo ocular, sendo utilizada transparência plástica contendo alvo com escala de $1 \mathrm{~cm}$; índice de vocalização desde a entrada no box de atordoamento até a calha de sangria; e presença de respiração rítmica após atordoamento do animal (área de vômito) até a calha de sangria; presença de reflexo de correção na postura (animal tentando adotar postura horizontal flexionando a cabeça) na área de vômito, durante o içamento e na calha de sangria.

Os dados foram submetidos ao teste de estatística não paramétrica Kruskal-Wallis, para o estudo do efeito dos principais dos tratamentos, sendo utilizado o software estatístico Minitab ${ }^{\circledR}$, versão 12.1 . Considerou-se o dia como bloco, e o animal, como unidade experimental.

\section{RESULTADOS E DISCUSSÃO}

Os resultados relativos à eficácia do primeiro disparo (Tabela 1) corroboram os obtidos por GALLO \& CARTES (2000) e GALLO et al., (2003), nos quais o 
Tabela 1 - Valores médios, desvio padrão e porcentagem das mensurações de eficácia da insensibilização dos diferentes sistemas de contenção utilizados.

\begin{tabular}{llll}
\hline Variável & \multicolumn{1}{c}{ Box 1} & \multicolumn{1}{c}{ Box 2} & Valor $\underline{\mathrm{P}}$ \\
\hline Numero de disparos & $1,2025^{\mathrm{a}} \pm 0,026$ & $1,0625^{\mathrm{b}} \pm 0,013$ & 0001 \\
Eficácia do $1^{\mathrm{o}}$ disparo & $0,84^{\mathrm{a}} \pm 0,367$ & $0,94^{\mathrm{b}} \pm 0,238$ & 0,0001 \\
& $(84 \%)$ & $(94 \%)$ & $(\mathrm{NS})$ \\
Vocalizações & $0,05 \pm 0,011$ & $0,0425 \pm 0,011$ & 0,001 \\
& $(5 \%)$ & $(4 \%)$ & \\
Respiração rítmica & $0,0275^{\mathrm{a}} \pm 0,008$ & $0,00^{\mathrm{b}}$ & (NS) \\
Reflexo de correção na postura & $(2,75 \%)$ & $(0 \%)$ & (NS) \\
Tentativa de fuga & $0,0225 \pm 0,007$ & $0,0225 \pm 0,007$ & $(2,25 \%)$ \\
\hline
\end{tabular}

${ }^{a}$ Valores com sobrescritos diferentes em uma mesma linha são estatisticamente diferentes de acordo com o teste de kruskal- wallis $(\mathrm{P}<0,001)$; (NS) = não significativo.

box automatizado apresentou eficácia de 94\% contra 84\% do box mecanizado. Entretanto, os dois sistemas de contenção apresentaram eficácia menor que 95\%, limite proposto por GRANDIN (1999).

Estudos realizados por GRANDIN (1998a; 1998b) e a H.S.A (1998) reportaram que um colaborador fadigado pode causar falhas na tentativa de acerto ao primeiro disparo; entretanto, nenhum efeito significativo do horário (manhã ou tarde), assim como do período (início ou final do turno de abate), foi encontrado no presente estudo, em relação às mensurações das tabelas 1 e 2 .

O treinamento e a capacitação dos colaboradores também podem contribuir para uma elevação da eficiência do primeiro disparo, como foi relatado no estudo de GALLO et al. (2003). No presente estudo, o operador não tinha recebido o curso de capacitação, o que pode ter contribuído para a obtenção de um índice de eficácia no primeiro disparo inferior a 95\%, como proposto por GRANDIN (1999).

Segundo GRANDIN (2000), em 1996, somente $30 \%$ das plantas frigoríficas dos Estados Unidos conseguiam atingir eficácia de 95\% no primeiro disparo. A mudança ocorreu em 1999, quando 90\% das plantas conseguiram obter resultados satisfatórios na insensibilização. Destaca-se que a melhora não foi somente resultado das mudanças na infraestrutura e capacitação, mas também foi de fundamental importância a constante vigilância da gerência das plantas frigoríficas.

O número de disparos necessários para proporcionar a insensibilização foi significativamente maior no box 1 (Tabela 1). Um maior número de disparos pode proporcionar prejuízos ao bem-estar do animal, que sente dor e tem seu nível de estresse aumentado (CIOCCA et al., 2006).

Tabela 2 - Valores médios, desvio padrão e porcentagem do número de disparos e distância em relação ao ponto ideal de insensibilização dos diferentes sistemas de contenção utilizados.

\begin{tabular}{|c|c|c|c|c|c|c|}
\hline Variável & Total & $\mathrm{N}$ & Box 1 & $\mathrm{~N}$ & Box2 & Valor p \\
\hline $1^{0}$ disparo & $\begin{array}{c}800 \\
(100 \%)\end{array}$ & $\begin{array}{c}400 \\
(100 \%)\end{array}$ & $2,357^{\mathrm{a}} \pm 1,246$ & $\begin{array}{c}400 \\
(100 \%)\end{array}$ & $1,246^{\mathrm{b}} \pm 0,918$ & 0,000 \\
\hline $2^{\underline{0}}$ disparo & $\begin{array}{c}88 \\
(11 \%)\end{array}$ & $\begin{array}{c}64 \\
(16 \%)\end{array}$ & $4,016^{\mathrm{a}} \pm 0,101$ & $\begin{array}{c}24 \\
(6 \%)\end{array}$ & $3,042^{\mathrm{b}} \pm 0,141$ & 0,000 \\
\hline $3^{\circ}$ disparo & $13(1,62 \%)$ & $\begin{array}{c}12 \\
(3 \%)\end{array}$ & 4,083 $\pm 0,193$ & $\begin{array}{c}1 \\
(0,25 \%)\end{array}$ & $4,000 \pm 0,000$ & 0,878 (NS) \\
\hline $4^{\circ}$ disparo & $\begin{array}{c}4 \\
(0,5 \%)\end{array}$ & $\begin{array}{c}4 \\
(1 \%)\end{array}$ & $4,500 \pm 0,289$ & 0 & - & NS \\
\hline $5^{\circ}$ disparo & $1(0,12 \%)$ & $\begin{array}{c}1 \\
(0,25 \%)\end{array}$ & $4,000 \pm 0,000$ & 0 & - & NS \\
\hline
\end{tabular}

${ }^{a}$ Valores com sobrescritos diferentes em uma mesma linha são estatisticamente diferentes de acordo com o teste kruskal- wallis (P<0,05).

Ciência Rural, v.40, n.8, ago, 2010. 
Os resultados do presente estudo corroboram os trabalhos de EWBANK et al. (1992), GRANDIN (1993) e GALLO et al. (2003), os quais verificaram que pode ser melhorada a eficácia da insensibilização, mediante o uso de mecanismo para a fixação da cabeça, porém o sitema de contenção deve ser desenhado de tal maneira que o animal entre de forma voluntária, que não possa girar dentro do box e que seja insensibilizado imediatamente após a imobilização. No Brasil, não existe regulamentação para a utilização do box de atordoamento com contenção adequada da cabeça, porém algumas indústrias já fazem uso desse equipamento, devido a pressões de seus clientes voltadas para com o bem-estar animal.

Apesar de a utilização do box automatizado com contenção de cabeça melhorar a exatidão do atordoamento, maior estresse pode ocorrer se o sistema for mal desenhado ou utilizado inadequadamente. EWBANK et al. (1992) indicam que o animal deve ser atordoado dentro de cinco segundos depois de a cabeça estar fixada.

O responsável pelo atordoamento tem um papel fundamental na insensibilização do animal e deve saber o local, a posição e a direção corretos dos disparos (H.S.A., 1998; FINNIE, 1997). GALLO \& CARTES (2000) observaram que o sucesso da utilização do box automatizado melhorou significativamente após a capacitação dos funcionários.

A H.S.A. (1998) pondera que o local do disparo deve estar em um raio de $2 \mathrm{~cm}$ do local ideal; entretanto, GRANDIN (2002) considera que, no atordoamento com pistola penetrante, a efetividade ocorre até $6 \mathrm{~cm}$ do local ideal, visto que o sucesso do atordoamento dependerá mais da direção e força do disparo. Os resultados obtidos por GALLO et al. (2003) corroboram o preconizado por GRANDIN (2002), visto que, apesar de ter sido encontrada uma ampla distribuição dos locais de disparo na utilização do box automatizado (somente $58,3 \%$ dos disparos se encontravam a menos de $2 \mathrm{~cm}$ do local ideal), elevada porcentagem de bovinos apresentou sinais de insensibilização adequados.

Por outro lado, GREGORY (1994) relata que, quando o disparo dista a mais de $2 \mathrm{~cm}$ do local ideal, ocorrem falhas na insensibilização. H.S.A (1998) demonstrou que, se o local do disparo estiver 4-6 cm do local ideal, a eficácia da insensibilização será de $60 \%$.

O presente estudo corrobora os resultados de GREGORY (1994) e a H.S.A. (1998), visto que 31,6\% dos disparos localizados até 2cm do local ideal e 40,5\% (4,1 a $6 \mathrm{~cm}$ do local ideal) foram obtidos com a utilização dos box 1, enquanto que, com a utilização do box 2 ,
60,5\% dos disparos localizados até $2 \mathrm{~cm}$ do local ideal e $11 \%$ (4.1 a $6 \mathrm{~cm}$ do local ideal) foram obtidos.

De acordo com a H.S.A. (1998), PRATA \& FUKUDA (2001) e GRANDIN (1999), a respiração rítmica é o sinal mais evidente de uma insensibilização inadequada e indicativa de que o animal está sensível. No presente estudo (Tabela 1), visualiza-se que 2,75\% dos animais atordoados no box 1 apresentaram reflexos de respiração, não atendendo ao limite de 0,2\% proposto por GRANDIN (1999); por outro lado, a utilização do box 2 proporcionou ausência desse indicador de sensibilidade

A vocalização é um sinal de incômodo ou dor, pois bovinos raras vezes vocalizam durante o manejo dos currais ao box de atordoamento ou quando se encontram nele. Considera-se aceitável até 3\% de vocalização durante esses manejos (GRANDIN, 2000, 2001).

No presente estudo, da entrada do box de atordoamento até o momento da sangria, (Tabela 1), observou-se que $5 \%$ dos bovinos insensibilizados no box 1 e 4\% no box 2 vocalizaram, entretanto essa variação não foi significativa estatisticamente. GALLO et al. (2003) demonstrou que a frequência de vocalização diminuiu significativamente com a utilização do box de contenção automatizado e desapareceu depois da capacitação dos magarefes.

O reflexo na correção da postura após atordoamento ou o levantamento da cabeça na calha de sangria são sinais claros de um processo de insensibilização ineficaz e estaria entre os mais confiáveis itens de avaliação de sensibilidade (GRANDIN, 1998a). Observa-se, na tabela 1, que 5 e 2,25\% dos animais insensibilizados no box 1 e 2 apresentaram reflexo de correção. Embora não tenham sido encontradas diferenças significativas entre os sistemas de contenção estudados, os dados não estão de acordo com o preconizado por GRANDIN (1999), que estabelece um limite máximo de 0,2\% para esse indicador de sensibilidade.

A presença dos sinais de sensibilidade observados isoladamente não pode ser conclusiva a respeito da sensibilidade dos animais (BLACKMORE \& DELANY, 1988). A presença de vários sinais de sensibilidade em alta porcentagem reflete, em geral, que há uma baixa eficácia em provocar a inconsciência plena do animal, e o processo não está cumprindo o objetivo primordial de evitar o sofrimento dos bovinos destinados à produção de carne (GRANDIN, 1994; 1998; H.S.A., 1998). 


\section{CONCLUSÃO}

Os resultados do presente estudo demonstraram claramente que a implementação do box de atordoamento automatizado (com fixação da cabeça) permitiu uma melhoria na eficácia da insensibilização dos bovinos comparativamente ao box convencional (sem fixação de cabeça). Esse sistema proporcionou menor número de disparos para induzir insensibilidade, maior eficácia no primeiro disparo, menor índice de respiração rítmica e disparos mais próximos do ponto ideal, o que resultou em uma elevação do bem-estar ao animal. Destaca-se que, embora o box automatizado tenha apresentado melhor eficácia, melhores índices de desempenho serão obtidos após a capacitação do operador para esse equipamento.

\section{REFERÊNCIAS}

BLACKMORE D.K.; DELANY, M.W. Cap. 4: Assessment of insensibility, p.23-27; Cap.8: Percussive stunning, p.55-71; Cap.11: Choosing an appropriate method of slaughter, p.95100. Slaughter of stock: A practical review and guide. In: Veterinary Continuing Education, Massey University, Palmerston North New Zeland, 1988.

CIOCCA, J.R.P. et al. O treinamento dos funcionários de plantas frigoríficas melhora a eficîência do atordoamento de bovinos. In: CONGRESSO INTERNACIONAL DE CONCEITOS EM BEM-ESTAR ANIMAL, 2006, Rio de Janeiro-RJ. Anais... Rio de Janeiro-RJ: WSPA, 2006. V.1, p.1-1.

EIKELENBOOM, G. Some aspects of captive bolt pistol stunning in ruminants. Stunning of animals for slaughter. Leiden, Netherlands: Martinus Niijhoff Publishers, 1983. 7p.

EWBANK, R. et al. Reaction of cattle to head restraint at stunning: A practical dilemma. Anim Welf, v.1, p.55-63, 1992.

FINNIE, J.W. Brain damage caused by a captive bolt pistol. J Comp Pathol, v.3, p.253-258, 1993.

FINNIE, J. Traumatic head injury in ruminant livestock. Aust Vet J, v.75, p.204-208, 1997.

GALLO, C.; CARTES, M. Insensibilización en bovinos: evaluación de la eficacia en el uso de la pistola de proyectil retenido en 3 plantas de la X Región. In: CONGRESSO NACIONAL DE MEDICINA VETERINARIA, 11., 2000, Santiago. Anais... Santiago, Chile, 2000. p.24-27.

GALLO, C. et al. Mejoras en la insensibilización de bovinos con pistola neumática de proyectil retenido tras cambios de equipamiento y capacitación del personal. Arch Med Vet, v.35, n.2, p.159-170, 2003.

GRANDIN, T. Behavior of slaughter plants and auction employees towards animals. Anthrozoos, v.1, p.205-213, 1988.
GRANDIN, T. Handling and welfare of livestock in slaughter plants. In: CABI International. Livestock handling and transport. Oxfordshire, UK: Department of Animal Science, Colorado State University, Fort Collins, Colorado, U.S.A., 1993. Cap.19, p.289-307.

GRANDIN, T. Farm animal welfare during handling, transport, and slaughter. J Am Vet Med Assoc, v.204, n.3, p.372-377, 1994.

GRANDIN, T. Animal Welfare in Slaughter Plants. 1996. 4p. Disponível em: <http://www.grandin.com/welfare/ general.session.html>. Acesso em: 26 jun. 2010.

GRANDIN, T. Objective scoring for animal handling and stunning practices in slaughter plants. J Am Vet Med Assoc, v.212, p.36-39, 1998a.

GRANDIN, T. Solving livestock handling problems in slaughter plants. In: GREGORY, N. et al. Animal welfare and meat science. Oxfordshire, UK: CABI Publishing, 1998b. Cap.3, p.42-45.

GRANDIN, T. Buenas prácticas de trabajo para el manejo e insensibilización de animales. 1999. Disponível em: <http://www.grandin.com/spanish/Buenas.practicas.html>. Acesso em: 01 jul. 2010.

GRANDIN, T. Effect of animal welfare audits of slaughter plants by a major fast food company on cattle handling and stunning practices. J Am Vet Med Assoc, v.216, p.848-851, 2000 .

GRANDIN, T. Cattle vocalizations are associated with handling and equipment problems at beef slaughter plants. Appl Anim Beh Sci, n.71, p.191-201, 2001.

GRANDIN, T. Return to sensibility problems after penetrating captive bolt stunning of cattle in commercial beef slaughter plants. J Am Vet Med Assoc, v.221, p.1258-1261, 2002.

GRANDIN, T. Proper cattle restraint for stunning. Updated April 2010. Disponível em: <http://www.grandin.com/ humane/restrain.slaughter.html>. Acesso em: 26 jun. 2010.

GREGORY, N. Preslaughter handling, stunning and slaughter. Meat Science, v.36, p.45-56, 1994.

GREGORY, N.G. et al. Depth of concussion in cattle shot by penetrating captive bolt. Meat Science, v.77, p.499-503, 2007 .

HEDRICK, H.B. et al. Principles of meat science. 3.ed. San Francisco, CA: B.S. Schweigert Freeman, 1994. 288p.

HUMANE SLAUGHTER ASSOCIATION (H.S.A.). Captive bolt stunning of livestock. 2.ed Wheathampstead, Hertfordshire, 1998. 14p. Disponível em: <http://www.hsa.org.uk/> Acesso em: 26 jun. 2010. Copyright (c) WWW.hsa.org.uk Charity n.209563.

NEVES, J.E.G. Influências de métodos de abate no bemestar e na qualidade da carne de bovinos. 2008. $70 \mathrm{f}$. (Dissertação de mestrado em Zootecnia) - Universidade Estadual Paulista “Julio de Mesquita Filho” Faculdade de Ciências Agrárias 
e Veterinárias Câmpus de Jaboticabal. Disponível em: <http:// www.fcav.unesp.br/download/pgtrabs/zoo/m/3486.pdf>. Acesso em: 20 jun. 2009.

PRATA L.F.; FUKUDA T.R. Fundamentos de higiene e inspeção de carnes. Jaboticabal: FUNEP, 2001. 326p.

ROÇA, R.O. Abate humanitário melhora a carne: bem-estar do animal na hora do abate influencia na qualidade do produto.
Revista do açougueiro e frigorífico, v.5, n.42, p.28-30, 1999.

ROÇA, R.O. Abate humanitário de bovinos. In: CONFERÊNCIA VIRTUAL GLOBAL SOBRE PRODUÇÃO ORGÂNICA DE BOVINOS DE CORTE, 2002. Anais... [S.l.: s.n.]. Disponível em: <www. cpap. embrapa. br/agencia/congressovirtual/pdf/ portugues/ 02pt03.pdf>. Acesso em: 24 jun. 2010. doi: <http:/ /dx.doi.org/10.1016/S0022-474X(00)00016-3. 